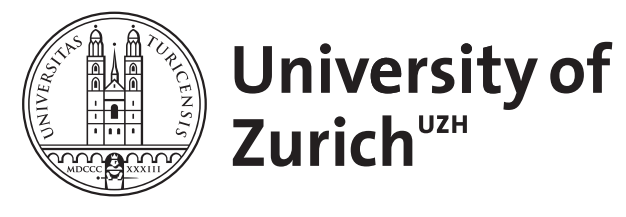

\title{
Impfungen in der Schwangerschaft
}

\author{
Stahel, Michèle
}

\begin{abstract}
Impfungen vor und während der Schwangerschaft kommt eine wichtige Bedeutung zu. Viele Probleme könnten bei konsequenter Impfstrategie verhindert werden. Zwei Impfungen werden zurzeit routinemässig in jeder Schwangerschaft empfohlen. Einerseits die saisonale Grippeimpfung, die vor allem die Mutter vor der Influenza-Infektion während der Schwangerschaft schützen soll, da Schwangere häufiger schwere Verläufe oder Komplikationen aufweisen, und andererseits die Pertussisimpfung, die dem Neugeborenen in den ersten Lebenswochen nachweislich einen Schutz gibt.
\end{abstract}

DOI: https://doi.org/10.1024/1661-8157/a003312

Other titles: Vaccination During Pregnancy

Posted at the Zurich Open Repository and Archive, University of Zurich ZORA URL: https://doi.org/10.5167/uzh-179154

Journal Article

Published Version

Originally published at:

Stahel, Michèle (2019). Impfungen in der Schwangerschaft. Praxis, 108(13):859-862.

DOI: https://doi.org/10.1024/1661-8157/a003312 


\title{
Impfungen in der Schwangerschaft
}

\author{
Vaccination During Pregnancy
}

\author{
Michèle Stahel \\ Klinik für Geburtshilfe, Frauenklinik Zürich
}

\begin{abstract}
Zusammenfassung: Impfungen vor und während der Schwangerschaft kommt eine wichtige Bedeutung zu. Viele Probleme könnten bei konsequenter Impfstrategie verhindert werden. Zwei Impfungen werden zurzeit routinemässig in jeder Schwangerschaft empfohlen. Einerseits die saisonale Grippeimpfung, die vor allem die Mutter vor der Influenza-Infektion während der Schwangerschaft schützen soll, da Schwangere häufiger schwere Verläufe oder Komplikationen aufweisen, und andererseits die Pertussisimpfung, die dem Neugeborenen in den ersten Lebenswochen nachweislich einen Schutz gibt.
\end{abstract}

Schlüsselwörter: Impfung, Schwangerschaft, Grippeimpfung, Pertussisimpfung

\begin{abstract}
Vaccinations administered to women during pregnancy can provide protection against serious infectious diseases for the mother, for the newborn, or both. Two vaccines are routinely recommended during pregnancy: Influenza and Pertussis. While the influenza vaccine protects mainly the pregnant mother, who is at an increased risk for severe affection, the pertussis vaccine is an effective protection to infants who are at particularly high risk of pertussis complications and mortality in the first three months of life.
\end{abstract}

Keywords: Vaccination, pregnancy, influenza vaccination, pertussis vaccination

\begin{abstract}
Résumé: Les vaccins avant et durant la grossesse deviennent de plus en plus importants pour éviter de nombreux problèmes pendant et après la grossesse. Deux vaccins sont actuellement conseillés à toutes les femmes enceintes. Le vaccin contre la grippe protège essentiellement la femme enceinte plus fréquemment atteinte de complications tandis que le vaccin contre la coqueluche est une stratégie efficace de protection du nouveau-né pendant les premières semaines de la vie.
\end{abstract}

Mots-clés: Vaccination, grossesse, vaccination contre la grippe, vaccination contre la coqueluche

Das Thema Impfungen, und insbesondere Impfungen in der Schwangerschaft, wird aktuell viel, unter anderem auch in der Laienpresse, diskutiert. Es scheint, dass kaum ein Thema mehr Skepsis hervorruft als Impfungen. Einerseits haben sich die Impfempfehlungen in den letzten Jahren stark verändert und andererseits kennen viele, auch Ärzte, die Probleme und Komplikationen fast vergessener Krankheiten nicht mehr. Diese haben ihren Schrecken verloren, und Sorgen über mögliche Nebenwirkungen und häufig falsche Ängste sind weit verbreitet.

Es ist jedoch von grosser Bedeutung, dass Ärzte und auch Patientinnen gut über die Wichtigkeit von Impfungen vor und insbesondere auch während der Schwangerschaft aufgeklärt sind [1-3]. Es geht um den Schutz der Mutter, des Fetus und des Neugeborenen. Den Gynäkologen kommt hierbei eine wichtige Rolle bei der Beratung betreffend Impfungen zu.

\section{Einteilung der Impfungen}

Prinzipiell können Impfungen im Hinblick auf eine Schwangerschaft in vier Gruppen eingeteilt werden [4]:

1. Impfungen, die vor einer Schwangerschaft empfohlen werden.

2. Impfungen, die während der Schwangerschaft empfohlen werden.

3. Impfungen, die bei Bedarf während der Schwangerschaft gemacht werden dürfen.

4. Impfungen, die in der Schwangerschaft kontraindiziert sind.

In diesem Artikel wird ein besonderes Augenmerk auf Punkt 2 gelegt. 


\section{Impfungen, die vor einer Schwangerschaft empfohlen werden}

Es ist wichtig, dass bei Frauen, die eine Schwangerschaft planen oder schwanger werden könnten, die Impfungen kontrolliert werden. Häufig ist der Besuch beim Gynäkologen der einzige Arztbesuch einer jungen Frau, weshalb diesen Ärzten eine besondere Rolle bei der Impfberatung zukommt. Idealerweise werden Frauen vor einer geplanten Schwangerschaft kontrolliert und gegebenenfalls (nach)geimpft.

Die empfohlenen Impfungen entsprechen den allgemeinen Impfempfehlungen des Bundesamts für Gesundheit (BAG).

Neben Hepatitis A und B (kann kombiniert werden), Diphterie und Tetanus und natürlich den Impfungen gegen humane Papillomaviren (HPV) sind insbesondere die Masern/Mumps- und Röteln-Impfungen sehr wichtig (zum Beispiel als Kombinationsimpfung MMR mit zwei Dosen). Diese drei typischen Kinderkrankheiten sind zum Glück heute selten geworden, und doch lohnt es sich, sich daran zu erinnern, weshalb sie so gefürchtet waren und es wichtig ist, die Frauen und die Ungeborenen davor zu schützen.

Rubella verläuft häufig unbemerkt, allerdings haben Schwangeren, ein erhöhtes Risiko, einen Abort zu erleiden, oder aber die Krankheit führt zu schweren Fehlbildungen beim Fetus, insbesondere am Herzen, am Auge bis zur Blindheit, am Ohr mit Taubheit oder im Gehirn in Form von geistiger Behinderung. Das Risiko ist im 1. Trimenon besonders hoch und nimmt ab der 20. Woche ab [5].

Eine Maserninfektion in der Schwangerschaft birgt ein erhöhtes Risiko für Aborte, Früh- oder Totgeburten. Es kommt nicht zu Missbildungen beim Fetus. Bei Säuglingen führen Masern oft zu Komplikationen und zu vermehrten und verlängerten Hospitalisationen. Maserninfektionen bei Schwangeren können zu schweren Pneumonien oder einer Meningitis führen.

Eine Infektion mit Mumps in der Schwangerschaft kann das Risiko eines Aborts erhöhen [5].

Varizellenimpfungen sollten durchgeführt werden bei Frauen, die die Varizellen nicht in der Kindheit durchgemacht haben und noch nicht geimpft wurden, denn Varizellen verursachen bei Erwachsenen und besonders bei Schwangeren häufiger Komplikationen als bei Kindern (z.B. Pneumonie, bakterielle Superinfektionen, Meningitis oder Enzephalitis). Bei Infektion in der ersten Schwangerschaftshälfte können sie beim Fetus Fehlbildungen mit schwerer Schädigung der Haut, Knochen, Augen oder des Gehirns verursachen [5].

Peripartal kann eine Varizelleninfektion der Mutter beim Neugeborenen die gleiche Krankheit verursachen, was mit einer erhöhten Mortalität (bis zu 30\%) des Neugeborenen verbunden ist.

Ausserdem wird die FSME-Impfung insbesondere in Endemiegebieten wie zum Beispiel dem Kanton Zürich empfohlen.
Je nach Saison lohnt sich eine Grippeimpfung bereits vor einer geplanten Schwangerschaft. Zudem können spezielle Impfungen wie z.B. Gelbfieberimpfung je nach Lebensstil (Reisen) indiziert sein.

Bei der Verabreichung von Lebendimpfstoffen sollte sichergestellt sein, dass die Frau nicht bereits schwanger ist. Allerdings ist eine akzidentelle Impfung kein Grund für einen Schwangerschaftsabbruch.

\section{Empfohlene Impfungen während einer Schwangerschaft}

Dem Impfen in der Schwangerschaft kommt eine ganz besondere Rolle zu. Einerseits soll die Impfung die Mutter und den Fetus schützen, beziehungsweise dann dem Neugeborenen einen Nestschutz geben.

Untersuchungen haben gezeigt, dass Schwangere für gewisse Infektionskrankheiten eine erhöhte Anfälligkeit haben, andere Infektionskrankheiten zeigen häufig einen schwereren Verlauf bei Schwangeren. Die Grippepandemie im Jahr 2009 ist ein Beispiel aus der jüngeren Geschichte, die zeigt, dass Schwangere unverhältnismässig stärker betroffen sind von der Infektion [6]. Erklärt wird dies folgendermassen: Es kommt während der Schwangerschaft $\mathrm{zu}$ verschiedenen mechanischen und (patho-) physiologischen Veränderungen, wie zum Beispiel zu einem verminderten Lungenvolumen, erhöhter Herzfrequenz und immunologischen Adaptationsmechanismen.

Es ist wichtig zu wissen, dass gesunde Schwangere auf Impfungen genau gleich eine Immunantwort aufbauen können wie Nicht-Schwangere [7].

Zwei Impfungen werden zurzeit während der Schwangerschaft empfohlen:

a) Pertussis

Allen Schwangeren wird zurzeit empfohlen, die Impfung gegen Pertussis während der Schwangerschaft durchführen zu lassen.

Schwangere Frauen haben zwar kein erhöhtes Risiko für einen schweren Verlauf einer Pertussisinfektion, jedoch sind Neugeborene und Säuglinge betreffend einer solchen Infektion gefährdet und haben ein erhöhtes Risiko für Komplikationen. Insbesondere in den ersten Lebensmonaten ist die Infektion mit einer hohen Morbidität verbunden. Die häufigsten Infektionsquellen sind Familienmitglieder. Der Schutz der Pertussisimpfung ist nicht langanhaltend. Der schweizerische Impfplan empfiehlt den Impfbeginn im Alter von zwei Monaten. Ein guter Schutz vor Pertussis ist nach zwei Dosen zu erwarten, das heisst frühestens mit vier Monaten.

Da jedoch viele schwere Krankheitsverläufe in den ersten zwei bis drei Lebensmonaten auftreten, sollte die Mutter während der Schwangerschaft geimpft werden, und zwar unabhängig vom Impfstatus (das heisst, auch wenn sie bereits in der letzten Schwangerschaft vor z.B. einem Jahr geimpft wurde).

Die Mutter bildet nach der Impfung Antikörper, die über die Plazenta zum Fetus übertreten und so das Neuge- 
borene vor einer Infektion schützen [8]. Die Wirksamkeit beträgt über $90 \%$ [9]. Aktuell wird empfohlen, die Pertussisimpfung im zweiten Trimenon (zwischen der 13. und der 26. Schwangerschaftswoche) durchzuführen, damit auch Frühgeborene von der Impfung profitieren, spätestens aber zwei Wochen vor der Geburt. Auch bei 36 Schwangerschaftswochen verursacht eine Impfung noch einen genügenden Antiköperspiegel beim Kind [10]. Falls keine Impfung während der Schwangerschaft verabreicht wurde, kann diese im Wochenbett nachgeholt werden, falls die letzte Impfung der Mutter über zehn Jahre zurückliegt. Allerdings kann diese Impfung das Neugeborene in der vulnerablen Phase nicht sicher schützen, aber die Mutter wird somit nicht zur Infektionsquelle.

Aktuell gibt es keine Evidenz von negativen Auswirkungen einer Pertussisimpfung, weder auf den Schwangerschaftsverlauf noch auf die fetale Entwicklung.

b) Influenza

Veränderungen des Immunsystems während der Schwangerschaft bewirken eine vermehrte Anfälligkeit gegenüber Infektionen. Dies scheint aber bei der Grippeinfektion weniger zum Zug zu kommen. Vielmehr prädisponieren die physiologischen, hämodynamischen und respiratorischen Veränderungen in der Schwangerschaft für schwerere Verläufe. Mit zunehmendem Schwangerschaftsalter kommt es zu einer verminderten Lungenkapazität, einer erhöhten Herzfrequenz und einem erhöhten Schlagvolumen des Herzens. Dies macht die Schwangere anfälliger auf schwere Verläufe, was zu vermehrten Hospitalisationen und erhöhter Mortalität führt [6]. Eine Influenzainfektion kann bei schwangeren Frauen schwerwiegende Atemwegskomplikationen auslösen, vor allem während des 2. und 3. Trimenons, aber auch im ersten Monat nach der Entbindung.

Zudem wird diskutiert, dass eine Influenzainfektion $\mathrm{zu}$ Komplikationen wie Frühgeburtlichkeit oder intrauteriner Wachstumgretardierung führen kann [11]. In den ersten sechs Lebensmonaten des Kindes ist eine Infektion mit einer höheren Letalität des Kindes und einem erhöhten Risiko für komplizierte Verläufe verbunden [12].

Die Influenzaimpfung während der Schwangerschaft vermindert das Risiko einer Influenzainfektion der Schwangeren und damit das Komplikations- und Hospitalisationsrisiko signifikant. Auch der Fetus wird durch die Impfung geschützt [13]. Es kommt zu weniger Frühgeburten und weniger Wachstumsretardierungen [14]. Zudem vermindert die Impfung während der Schwangerschaft das Risiko einer Infektion bei Säuglingen in den ersten Lebensmonaten [15].

Gemäss dem aktuellen Wissensstand hat die Influenzaimpfung in der Schwangerschaft keine negativen Auswirkungen auf den Schwangerschaftsverlauf oder auf die Entwicklung des Fetus. Nebenwirkungen werden bei Schwangeren nicht häufiger beschrieben.

Von der World Health Organisation (WHO) wird die Impfung eindeutig empfohlen.

Optimal wäre es, die Schwangeren vor Ausbruch der Grippeepidemie zu impfen, also im Oktober oder Novem-

\section{Key messages}

- Imfungen vor und während der Schwangerschaft kommt eine zunehmende Bedeutung zu. Zudem hat ein Paradigmenwechsel stattgefunden. Früher galt es, Impfungen in der Schwangerschaft möglichst zu vermeiden, heute werden zwei Impfungen gemäss den aktuellen Empfehlungen während der Schwangerschaft empfohlen. Erstens die Pertussisimpfung, die einen passiven Schutz für das Neugeborene in den ersten Lebenswochen darstellt.

- Zweitens die Grippeimpfung, die einen aktiven Schutz für die Mutter während der Schwangerschaft und Stillzeit darstellt. Der Fetus und das Neugeborene werden so auch vor einer eventuellen Influenza geschützt.

- Nur Lebendimpfstoffe sind während der Schwangerschaft kontraindiziert. Versehentliche Impfungen sind aber kein Grund, einen Schwangerschaftsabbruch ins Auge zu fassen. Falls Impfungen vor oder während der Schwangerschaft vergessen wurden, sollten sie postpartal im Wochenbett nachgeholt werden.

\section{Lernfragen}

1. Bei der Pertussisimpfung ist folgende Aussage am zutreffendsten: (Einfachauswahl)

a) Eine Impfung während der Schwangerschaft sollte nur durchgeführt werden wenn ein ungenügender Impfschutz besteht .

b) Die Impfung gegen Pertussis sollte vor oder in der ersten Schwangerschaft durchgeführt werden

c) Es wird empfohlen, alle Frauen in jeder Schwangerschaft gegen Pertussis zu impfen

2. Eine Impfung gegen Influenza...(Einfachauswahl) a)...ist zu empfehlen in der Schwangerschaft, da die Schwangeren häufig schwere Krankheitsverläufe haben b)...führt in der Schwangerschaft häufiger zu Komplikationen und Nebenwirkungen.

c)...darf in der Schwangerschaft nur im zweiten oder dritten Trimenon verabreicht werden.

ber. Allerdings macht auch eine Nachholimpfung noch Sinn. Die Schwangere kann zu jedem Zeitpunkt der Schwangerschaft geimpft werden, auch im ersten Trimenon.

\section{Impfungen, die bei Bedarf während der Schwangerschaft gemacht werden dürfen}

Wenn Impfungen in der Schwangerschaft durchgeführt werden, sollte der Nutzen für Mutter und Kind das Risiko einer Impfung überwiegen [3]. Dies ist ganz klar gegeben für die beiden Impfungen Influenza und Pertussis.

Alle ausser Lebendimpfstoffe können aber prinzipiell in der Schwangerschaft verabreicht werden, wenn es indiziert 
ist. So zum Beispiel die FSME-, Hepatitis-A- und B-Impfung. Falls eine Reise in ein Polio-gefährdetes Gebiet geplant ist, darf die Impfung mit dem inaktivierten, parenteralen Impfstoff Poliorix ${ }^{\circledR}$ oder einem der Kombinationsimpfstoffe Reavix ${ }^{\circledast}$ oder Boostrix Polio ${ }^{\circledR}$ verabreicht werden. Auch Pneumokokken- oder Haemophilus-influenzaeImpfungen können unter bestimmten Umständen indiziert sein.

\section{Impfungen, die in der Schwangerschaft kontraindiziert sind}

Lebendimpfstoffe sind während der Schwangerschaft kontraindiziert. Sie haben rein theoretisch ein potenzielles Risiko für eine fetale Infektion. Obwohl unzählige Publikationen zeigen, dass eine akzidentelle Impfung gegen Röteln im ersten Trimenon nicht zu einer Rubeolenembryopathie führt, sind deshalb die $\mathrm{MMR}^{\circledR}$-Impfung und auch die anderen Lebendimpfstoff-Impfungen in der Schwangerschaft kontraindiziert. Nach einer solchen Impfung wird eine sichere Verhütung für vier Wochen empfohlen [3].

Akzidentelle Impfungen in der Schwangerschaft sind keine Indikation für einen Schwangerschaftsabbruch [16].

Falls es vor einer Schwangerschaft verpasst wurde, sollten bei allen Frauen die fehlenden Impfungen nachgeholt werden. Kein Impfstoff ist während der Stillzeit kontraindiziert.

\section{Bibliografie}

1. Commission fédérale pour les vaccinations (CFV), Office fédéral de la santé publique OFSP. 2018. https://www.bag.admin.ch/ bag/fr/home/das-bag/organisation/ausserparlamentarischekommissionen/eidgenoessische-kommission-fuer-impf fragen-ekif.html, letzter Zugriff: 16.07.2019.

2. Mueller Y, Eberhardt CS, Diana A, Martinez De Tejada B: Recommendations for the vaccination of pregnant women: a booster injection. Rev Med Suisse 2016;12:1708-1712.

3. Munoz FM, Jamieson DJ: Maternal Immunization. Obstet Gynecol 2019; 133: 739-753.

4. Zimmermann R: Handbuch Geburtshilfe. Zürich; Eigenverlag Verein zur Förderung der Klinik für Geburtshilfe am USZ: 2018.
5. BAG: Empfohlene Impfungen für Frauen vor, während und nach der Schwangerschaft. Bern: 2017. https://www.bag. admin.ch/bag/de/home/das-bag/publikationen/broschueren/ publikationen-uebertragbare-krankheiten/empf-impffrauen-schwanger.html; letzter Zugriff: 16.07.2019.

6. Kourtis AP, Read JS, Jamieson DJ: Pregnancy and infection. N Engl J Med 2014;371: 1077.

7. Chu HY, Englund JA: Maternal immunization. Birth Defects Res 2017; 109: 379-386

8. Englund JA: The influence of maternal immunization on infant immune responses. J Comp Pathol 2007; 137: 16-19.

9. Amirthalingam G, Andrews N, Campbell H, et al.: Effectiveness of maternal pertussis vaccination in England: an observational study. Lancet 2014; 384: 1521-1528.

10. Abraham C, Pichichero M, Eisenberg J, Singh S: Third-trimester maternal vaccination against pertussis and pertussis antibody concentrations. Obstet Gynecol 2018;131: 364-369.

11. Naresh A, Fisher BM, Hoppe KK, et al.: A multicenter cohort study of pregnancy outcomes among women with laboratoryconfirmed H1N1 influenza. J Perinatol 2013; 33: 939-943.

12. Zaman K, Roy E, Arifeen SE, et al.: Effectiveness of maternal influenza immunization in mothers and infants. N Engl J Med 2008; 359: 1555-1564.

13. Poehling KA, Szilagyi PG, Staat MA, et al.: Impact of maternal immunization on influenza hospitalizations in infants. Am J Obstet Gynecol 2011; 204: 141-148.

14. Nunes MC, Aqil AR, Omer SB, Madhi SA: The effects of influenza vaccination during pregnancy on birth outcomes: A systematic review and meta-analysis. Am J Perinatol 2016; 33: 1104-1114.

15. Madhi SA, Nunes MC, Cutland CL: Influenza vaccination of pregnant women and protection of their infants. N Engl J Med 2014; 371: 918-931.

16. Keller-Stanislawski B, Englund JA, Kang G, et al.: Safety of immunization during pregnancy: a review of the evidence of selected inactivated and live attenuated vaccines. Vaccine 2014; 32: 7057-7064.

Manuskript eingereicht: 21.05.2019

Manuskript akzeptiert: 11.06.2019

Interessenkonflikt: Die Autorin erklärt, dass keine Interessenskonflikte bestehen.

\section{Dr. Michèle Stahel}

Oberärztin Klinik für Geburtshilfe

Schwerpunkttitel fetomaternale Medizin

Frauenklinikstrasse 10

8091 Zürich

michele.stahel@usz.ch

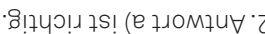

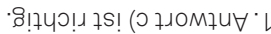

иәภельләา иәр nz иәжомұи 